\title{
La Charla Motivacional; una Estrategia para Abordar el Desconocimiento de Factores de Riesgo Ergonómico en un Supermercado Chileno
}

\author{
THE MOTIVATIONAL SPEECH: A STRATEGY TO ADDRESS THE LACK OF UNAWARENESS ERGONOMIC RISK \\ FACTORS IN A CHILEAN SUPERMARKET
}

Pablo E. Aliaga', Javier I. Villarroel', Natalia D. Cossio ${ }^{1}$

1. Universidad Bernardo O'Higgins Facultad de Salud, Recreación y Deporte, Santiago, Chile.

\section{RESUMEN}

Los factores protectores y ergonómicos determinan la salud de la población trabajadora. Los factores de riesgo ergonómicos se enfocan a trastornos músculo-esqueléticos, lesiones músculo-esqueléticos, posturas forzadas, mala alimentación, obesidad y sedentarismo; y, los factores protectores se enfocan en promocionar estilos de vida saludables. Este proyecto se realizó utilizando la metodología de investigación de acción participativa. La muestra intencionada correspondió a 30 trabajadores de un supermercado de Santiago. El objetivo principal del estudio fue educar a través de charlas motivacionales sobre los factores ergonómicos y protectores. En el estudio se realizó una encuesta, previamente validad por expertos, para verificar el conocimiento y percepción de los participantes. Se descubrió que el $80 \%$ de los empleados tenía dolor físico; un 77\%, estrés producto de su trabajo; un 30\% de los trabajadores tenía el conocimiento de cómo adoptar una postura correcta antes de la realización de las charlas, percepción que aumentó a un $83 \%$ de conocimiento adquirido después de la realización de las charlas. Se logró apreciar una adecuada interacción entre las charlas motivacionales, la promoción de factores protectores y la información sobre riesgo ergonómico.

(Aliaga P, Villarroel J, Cossio N, 2016. La Charla Motivacional; una Estrategia para Abordar el Desconocimiento de Factores de Riesgo Ergonómico en un Supermercado Chileno. Cienc Trab. May-Ago; 18 [56]: 106-109).

Palabras claves: FACTORES ERGONÓMICOS, FACTORES PROTECTORES, CHARLA MOTIVACIONAL, PROMOCIÓN DE SALUD.

\section{ABSTRACT}

Protective and ergonomic factors determine the health of the working population. Ergonomic risk factors are focused on muscle-skeletal disorders, muscle-skeletal injuries, awkward postures, poor diet, obesity, sedentary and protective factors are focused on promoting healthy lifestyles. This project was conducted through participatory action research methodology. The sample consisted of 30 employees of a supermarket in Santiago. The main objective of the study was to educate through motivational talks on ergonomic and protective factors. The study used a survey, previously validated by experts to verify the knowledge and perception of the participants. It was found that $80 \%$ of employees had physical pain, stress $77 \%$ product of their work, 30\% of workers had knowledge of what was correct posture before the completion of the talks, increased perception to $83 \%$ of knowledge gained after the completion of the talks. It was possible to appreciate a proper interaction between the motivational talks, promoting protective factors and ergonomic risk information.

Keywords: ERGONOMIC FACTORS, PROTECTIVE FACTORS, MOTIVATIONAL SPEECH, HEALTH PROMOTION.

\section{INTRODUCCIÓN}

El presente estudio pretende ser un aporte para la creación de estrategias de prevención ergonómicas, puesto que da a conocer los factores protectores y de riesgo de la población de estudio, indicando dónde es adecuado destinar recursos para lograr intervenciones eficaces. ${ }^{1}$

\section{Correspondencia / Correspondence:}

Pablo Aliaga

Sánchez Fontecilla 4680

código postal: 8320000

Tel.: 7897473, Tel. celular: 68494943,

e-mail: aliaga_arenas@hotmail.com

Recibido: 03 de Diciembre de 2015 / Aceptado 15 de Marzo de 2016
El trabajo ha sido históricamente un riesgo para la salud. Las condiciones laborales han supuesto habitualmente una amenaza a la salud debido a los accidentes y enfermedades relacionadas con los factores de riesgo ergonómicos. ${ }^{2}$ La Organización Mundial de la Salud (OMS), define riesgo ergonómico como cualquier rasgo, característica o exposición de un individuo que aumente su probabilidad de sufrir una enfermedad o lesión; de acuerdo a la definición se pueden plantear distintas estrategias para prevenir los factores de riesgo que están propensos a sufrir los trabajadores. Los factores protectores son la expresión opuesta de los factores de riesgo, son factores que funcionan en la promoción de conductas positivas. El desconocimiento de los factores protectores es un factor de riesgo en sí mismo. ${ }^{1}$

Las preguntas del presente estudio fueron: ¿Cuáles serán los principales factores de riesgo de salud laboral que afectan a los trabajadores de un supermercado?; ¿La charla motivacional será una estrategia para prevenir factores de riesgo ergonómico? 
Observamos empíricamente que los trabajadores desconocen los distintos factores de riesgo ergonómicos que pueden sufrir en su área laboral. ${ }^{2,3}$ Existe bibliografía orientada a explicar la morbilidad laboral ocasionada por la exposición a determinados riesgos existentes en el lugar de trabajo, como a ciertas características individuales que hace que unos trabajadores sean más vulnerables a la aparición de factores de riesgo ergonómicos, por la práctica de su actividad laboral. ${ }^{4}$

La creación de sistemas de información sobre los factores de riesgo de las patologías laborales y sobre sus características médicolaborales permitirá la aplicación de programas específicos de prevención que consigan reducir la incidencia y gravedad de los riesgos laborales. ${ }^{4}$

Chile ha experimentado durante las últimas décadas profundos cambios en procesos productivos con nuevas tecnologías e implementos. Evidenciándose nuevos factores de riesgos ergonómicos y psicosociales que han mostrado influencia en el desarrollo y persistencia del problema de salud del trabajador. ${ }^{5}$

La industrialización no solo afecta directamente a los trabajadores o empleados en las nuevas industrias, sino que además tiene un considerable impacto en el ambiente; las condiciones de trabajo peligrosas o no controladas son contraproducentes para la economía, la cultura y la salud de la región. ${ }^{6,7}$

Una inadecuada preparación de las condiciones organizacionales de un lugar de trabajo, junto con la "intensidad" o el nivel de actividad del trabajo y la ropa que se utilice, pueden originar situaciones de riesgo sutiles para la salud. ${ }^{8}$

Los factores de riesgo ergonómico se clasifican en dos grupos: el área psicosocial y el área física. ${ }^{8}$

En el área psicosocial, los trabajos son complejos y difíciles de entender, dado que representan el conjunto de las percepciones y experiencias del trabajador y abarcan muchos aspectos. ${ }^{9}$

El aspecto psicosocial en el trabajo adquiere relevancia, ya que los cambios en la organización tensionan al trabajador emocional y cognitivamente, aumentando la carga psíquica y mental. Los factores psicosociales de riesgo son factores probables de daño a la salud y pueden afectar tanto a la salud física como a la psicológica. ${ }^{10,11}$

En el área física observamos factores de riesgo ergonómicos como vibración corporal, movimiento de alcance, postura forzada, manipulación de cargas, movimiento repetitivo, trabajo sentado y trabajo de pie. Frente a las exigencias biomecánicas (postura, fuerza, movimiento), si no existe una adecuada recuperación biológica de los tejidos, este esfuerzo puede asociarse con el origen o la presencia de trastornos músculo-esqueléticos (TME); estos son la primera causa de baja relacionada con las condiciones de trabajo, generan una carga física que puede desencadenar cuadros reversibles como la fatiga, hasta generar una lesión irreversible. ${ }^{12}$

Los objetivos planteados para este estudio fueron: educar sobre los riesgos laborales de los participantes, diseñar un programa de promoción a través de salud laboral, implementar el programa diseñado en base a charlas motivacionales.

\section{METOdOLOGÍA}

El presente estudio corresponde a una investigación acción participativa en salud con aspectos cuantitativos y cualitativos. Se abordó un grupo de 30 participantes; la selección de ellos fue intencionada según factibilidad de la intervención educativa. Se seleccionó a todos los trabajadores de un supermercado de la comuna de La Reina de Santiago, sin distinción de género, en un rango de edad que fluctuaba entre 20 a 60 años, nivel socioeconómico medio bajo, con un ingreso mensual entre $90.000 \mathrm{y}$ 240.000 mil pesos.

Se excluyó a toda persona mayor de 60 años, además de trabajadores con nivel socioeconómico medio alto y alto.

Para valorar el componente descriptivo sobre riesgos ergonómicos se ejecutó una encuesta antes y después de la intervención, esta fue validada por expertos; kinesiólogos, metodólogos y bioestadísticos de la Universidad Bernardo O’Higgins, Santiago de Chile.

Para la promoción de salud se diseñó e implementó una intervención con charlas motivacionales sobre riesgos laborales, como el estrés, sobrecarga, malas posturas, mala alimentación, uso de calzado apropiado, sedentarismo, obesidad, entre otras.

Se realizó un seguimiento en el área laboral, realizando ejercicios de elongación, flexibilidad y educándolos para una eficaz técnica de trabajo.

Se consideraron tres posibles respuestas, que consistían en Sí, No, Regular, para analizar el grado de conocimiento que los participantes tenían respecto a los contenidos expuestos en las charlas antes y después de la intervención. La respuesta Sí consistía en que los trabajadores estaban informados en lo que consistían los factores de riesgo ergonómicos; el No consistía en que los trabajadores no tenían información sobre las distintas consecuencias que traen para su salud los factores de riesgo ergonómicos; $y$ Regular es cuando los trabajadores tienen una idea sobre la información impartida en las charlas, pero no eran capaces de aplicarlas por su cuenta.

El estudio contó con la aprobación del comité de ética de la Universidad Bernardo 0'Higgins.

Para calcular el índice de masa corporal (IMC), se utilizó una tabla de la OMS, la cual clasificaba a los trabajadores según su peso y estatura en normal, sobrepeso y obeso.

Tabla 1.

Índice de masa corporal.

\begin{tabular}{|ll} 
& IMC \\
Bajo Peso & Menor que 18.5 \\
Normal & Entre 18.5 y 24.9 \\
Sobrepeso & Entre 25 y 29.9 \\
Obesidad & Sobre o igual a 30 \\
\hline
\end{tabular}

Figura 1.

Conocimiento de factores protectores.

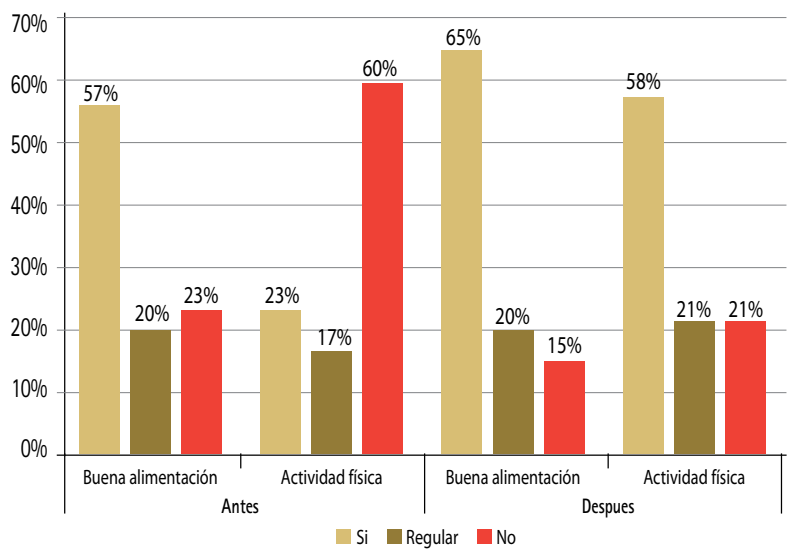




\section{RESULTADOS}

En la Figura 1 se analiza el conocimiento de los trabajadores sobre los 2 tipos de factores protectores, donde se incluyen buena alimentación y actividad física se observa que los trabajadores se sienten informados sobre lo que es una buena alimentación.

Figura 2.

Índice de masa corporal (IMC).

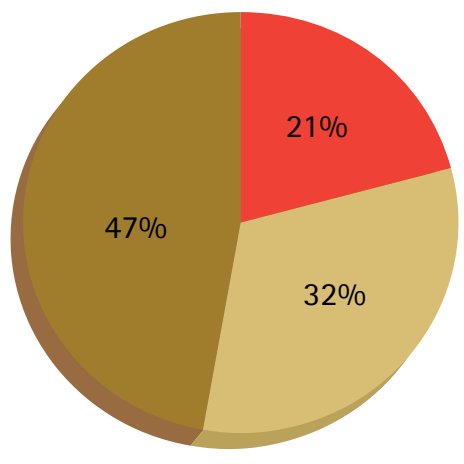

Normal Sobrepeso Obesidad

En la Figura 2 se analiza el IMC de los trabajadores de un supermercado, el cual se clasifica en normal, sobrepeso y obesidad.

Figura 3.

Comparación de conocimiento antes y después de las charlas motivacionales.

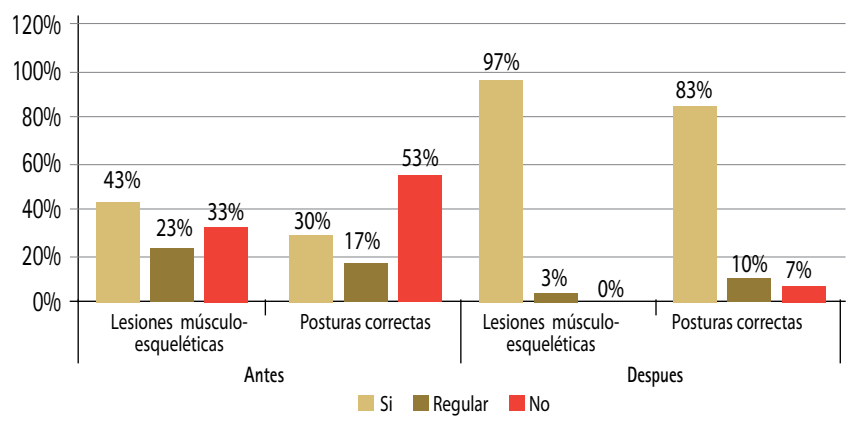

La Figura 3 muestra el conocimiento de los trabajadores antes y después de las charlas motivacionales realizadas por los estudiantes de kinesiología, en donde las variables son lesiones músculo-esqueléticas y postura correcta.

Figura 4.

Presencia de factores de riesgo ergonómicos.

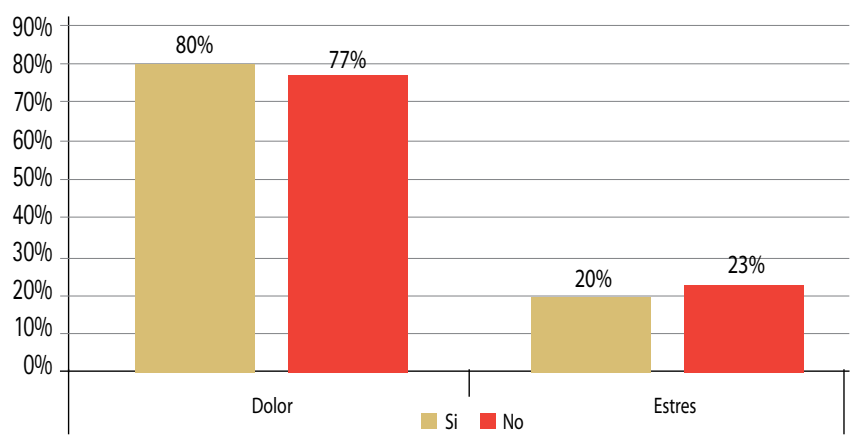

En la Figura 4 se muestra la presencia de dos factores de riesgo ergonómicos, en el ámbito físico en dolor y, en el psicológico, el estrés.

En la Figura 5 se muestra la percepción de los participantes respecto a tres variables de las charlas motivacionales: seguir asistiendo a las charlas; consideran que han adquirido conciencia; $\mathrm{y}$, utilizado el conocimiento aprendido sobre los factores de riesgo ergonómico y protectores.

Figura 5.

La percepción de los participantes respecto a las charlas motivacionales.

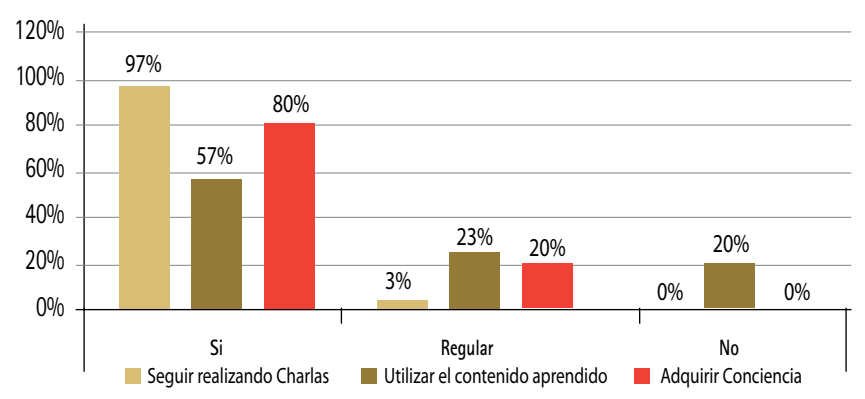

\section{DISCUSIÓN}

La ergonomía es el estudio científico del hombre en su trabajo; en particular, la aplicación de conceptos de anatomía, fisiología y psicología para resguardar su salud haciendo entender que el trabajo llegue a considerarse como más humano frente a los requerimientos físicos (postura, fuerza, movimiento). ${ }^{13-15}$

El estudio que utiliza como metodología la investigación acción participativa resuelve problemas cotidianos, para mejorar prácticas concretas de los participantes y sus conductas. Es una estrategia metodológica que consiste en propuestas educativas que buscan la autogestión comunitaria y la acción concertada e intersectorial en materia de salud pública. ${ }^{16-17}$

Los trabajadores presentan patologías derivadas de sus condiciones de trabajo cuando estas sobrepasan las capacidades de respuesta del individuo o cuando no existe una adecuada recuperación biológica de los tejidos, pudiendo existir una asociación con el origen o la presencia de trastornos músculo-esqueléticos y lesiones músculo-esqueléticas (LME), sobre todo si se presenta trabajo repetitivo, posturas inadecuadas, fatiga crónica y obesidad vinculada al sedentarismo. ${ }^{13,14}$

La postura se define como la alineación del cuerpo con una máxima eficiencia, tanto fisiológica como biomecánica, lo que reduce el estrés y las sobrecargas ejercidas sobre el sistema de sustentación, protegiendo al cuerpo contra traumas por los efectos de la gravedad. ${ }^{18}$ En la Figura 1 se observa que un 53\% de los trabajadores no sabe mantener una postura correcta en su entorno laboral y un 30\% que sí sabe, pero no la practica, ya que aproximadamente un $80 \%$ posee dolor muscular y un $77 \%$, estrés producto por su trabajo.

La presencia de malnutrición y sedentarismo es uno de los problemas de salud pública que presenta la población chilena en general. ${ }^{19}$ En este caso, un 57\% sí sabía lo que es una alimentación sana; se infiere que no practicaban estilos de vida saludable por sus altos índices de IMC y un 60\% no realizaba actividad física. En la Figura 2 se observa que el $68 \%$ de los trabajadores no 
tiene un peso acorde a su estatura; esto produce mayor sobrecarga articular en comparación a las personas que poseen un IMC normal, generando mayor probabilidad de LME.

En la Figura 3, un 43\% tenía conocimiento de qué era una LME previo a las charlas motivacionales. Cabe destacar que la gran mayoría de este porcentaje tenía dicho conocimiento por haber tenido una lesión y asistido a una consulta médica. Al finalizar el estudio, este número aumentó a un 97\%, sin haber ninguna lesión. La Primera encuesta nacional de empleo, trabajo, salud y calidad de vida de los trabajadores y trabajadoras de Chile (2009 a 2010), indica que el 72,1\% de los accidentes analizados en la encuesta se realizó atención médica y la mayor parte requirió realizar reposo con licencia médica y en un $27,9 \%$ no se efectuó, es decir, existe gran probabilidad de que un trabajador al lesionarse producto por su trabajo obtenga reposo con licencia médica. ${ }^{19}$

El concepto y desarrollo teórico de la promoción de la salud, en los lugares de trabajo, traslada a diversos escenarios laborales específicos para cada actividad, evidenciando la necesidad de brindar entornos saludables. ${ }^{20}$ De acuerdo a la percepción de los participantes, que se presenta en la Figura 5, un 97\% le gustaría que se siguieran impartiendo charlas motivacionales en el super- mercado, debido a que un 57\% ha utilizado el conocimiento aprendido y un $80 \%$ adquirió conciencia gracias a ellas. Para la Organización Mundial de Salud (OMS), la promoción de la salud en el lugar de trabajo incluye la realización de actividades en los lugares de trabajo ${ }^{20}$, evidenciando que las charlas motivacionales es una herramienta de promoción para distintas empresas en el área laboral.

En este estudio las charlas motivacionales demostraron ser una estrategia válida para el abordaje de la prevención de factores de riesgo ergonómico. También se pudo considerar y observar que los participantes se integraron activamente, dando su opinión de acuerdo a los temas abordados, demostrando interés respecto a darle continuidad a las charlas motivacionales. Creemos que la charla motivacional es una herramienta educativa que puede ser considerada por el profesional de la salud, ya que logra complementar al sujeto desde el punto de vista laboral y personal.

\section{Agradecimiento}

Se agradece a la profesora Lorena Llach, Eva Pérez, al director de carrera Jaime Ocaranza, al supermercado Santa Isabel de La Reina y a la facultad de salud de nuestra casa de estudios.

\section{REFERENCIAS}

1. Haquin $C$, Larraguibel $M$, Cabezas J. Factores protectores y de riesgo en salud mental en niños y adolescentes de la ciudad de Calama. Rev Chil Pediatr. 2004; 75(5):425-433.

2. Fagalde $M$, Solar J, Guerrero $M$, Atalah E. Factores de riesgo de enfermedades crónicas no transmisibles en funcionarios de una empresa de servicios financieros de la Región Metropolitana. Rev Méd Chile. 2005; 133:919-928.

3. Alvarado R, Pérez J, Saavedra N, Fuentealba C, Alarcón A, Marchetti N, Aranda $W$. Validación de un cuestionario para evaluar riesgos psicosociales en el ambiente laboral en Chile. Rev Med Chile. 2012; 140:1154-1163.

4. Ronda E, Roél J, Agudelo A, Berenguer S. Perfil epidemiológico de las enfermedades profesionales en Alicante. Med Segur Trab. 2009; 55(214):80-90.

5. Ratner $R$, Sabal J, Hernández $P$, Romero $D$, Atalah $D$. Estilos de vida y estado nutricional de trabajadores en empresas públicas y privadas de dos regiones de Chile. Rev Méd Chile. 2008;136: 1406-1414.

6. Robaina C, Fermin J. Aguirre R. ocupacional en paises en desarrollo. Rev Cubana Med Gen Integr. 2004; 133:919-928.

7. Breilh J. La determinación social de la salud como herramienta de ruptura hacia la nueva salud pública (salud colectiva). Rev Fac Nac Salud Pública. 2013; 31 (supl.1): S13-S27.

8. Muñoz C, Vanegas J, Marchetti N. Factores de riesgo ergonómico y su relación con dolor musculoesquelético de columna vertebral: basado en la primera encuesta nacional de condiciones de empleo, equidad, trabajo, salud y calidad de vida de los trabajadores y trabajadoras en Chile (ENETS). Med Segur Trab. 2012; 58(228):194-204.

9. Caraballo $Y$, Rafael A, Rivero A, Gerardo R, Barrios M. Riesgos Laborales en Trabajadores de Barberias y Peluquerías de Economía Informal. Cienc Trab. 2013; 15(46):18-23.

10. Carmo M, Chaves M, Cássia R, Almeida L, Aparecida I, Pedrão L. Exceso de trabajo y agravios mentales a los trabajadores de la salud. Rev Cubana Enfermer. 2010:26(1)52-64.

11. Organización Internacional del Trabajo-OIT. Identificación y reconocimiento de las enfermedades profesionales: Criterios para incluir enfermedades en la lista de enfermedades profesionales de la OIT. Reunión de expertos sobre la revisión de la lista de enfermedades profesionales. (Recomendación No. 194) [en línea]. Ginebra: OIT; 2009 [consultado may 2016]. Disponible en: http://www.ilo.org/ wcmsp5/groups/public/---ed_protect/---protrav/---safework/documents/ meetingdocument/wcms_116913.pdf

12. López $A$, León $F$, Holgado MS. Enfermedad profesional y médicos de familia. Med Segur Trab. 2010; 56(219):109-113.

13. Apud E, Meyer F. La importancia de la ergonomía para los profesionales de la salud. Cienc enferm. 2003;9(1):15-20.

14. Patricia $C$, Rodríguez M. Riesgo ergonómico en empresas artesanales del sector de la manufactura, Santander, Colombia. Med Segur Trab. 2013; 59(230):102-111.

15. Idaly A. Promoción de la salud en los lugares de trabajo: teoria y realidad. Med Segur Trab. 2010; 56(220):220-225.

16. Salgado AC. Investigación cualitativa: diseños, evaluación del rigor metodológico y retos. Liber. 2007; 13:71-78.

17. Perdomo G. La Investigación-Acción-Participante como Estrategia para la Capacitación y Evaluación en Educación Sanitaria. Cad Saude Públ. 1994; 10(3):331-338.

18. Palmer M. Fundamentos das técnicas de avaliação musculoesquelética. São Paulo: Guanabara-Koogan; 2000. 382 p.

19. Troncoso C Doepking C, Zúñiga B. Alimentación saludable en la formación de estudiantes de carreras del área pedagógica. Rev Chil Nutr. 2013; 40(1):43-47.

20.- Vallebuona C. Primera encuesta nacional de empleo, trabajo, salud y calidad de vida de los trabajadores y trabajadoras de Chile. ENETS 2009-2010. Informe interinstitucional [en linea]. Santiago de Chile: Gobierno de Chile; 2010 [consultado may 2016]. Disponible en: http://www.dt.gob.cl/documentacion/1612/ articles-99630_recurso_1.pdf

\section{Referencias complementarias}

Castellá JL. Calidad y Servicios de Prevención de Riesgos Laborales. Med Segur Trab. 2009; 55(216):10-11.

Vernaza-Pinzón P, Torres C. Dolor Músculo-Esquelético y su Asociación con Factores de Riesgo Ergonómicos, en Trabajadores Administrativos. Rev Salud Pública (Bogotá); 2005;7(3):317-326. 